\title{
Pacific
}

Journal of

Mathematics

\section{ON SECTIONS OF GENUS TWO LEFSCHETZ FIBRATIONS}

\author{
SINEM ÇELIK ONARAN
}




\title{
ON SECTIONS OF GENUS TWO LEFSCHETZ FIBRATIONS
}

\author{
SINEM ÇELIK ONARAN
}

\begin{abstract}
We find new relations in the mapping class group of a genus 2 surface with $n$ boundary components for $n=1, \ldots, 8$ that induce a genus 2 Lefschetz fibration $\mathbb{C P}^{2} \# 13 \overline{\mathbb{C P}}^{2} \rightarrow S^{2}$ with $n$ disjoint sections. As a consequence, we show any holomorphic genus 2 Lefschetz fibration without separating singular fibers admits a section.
\end{abstract}

\section{Introduction}

The study of Lefschetz fibrations is important in low-dimensional topology because of a close relationship between symplectic 4-manifolds and Lefschetz fibrations, [Donaldson 1999; Gompf and Stipsicz 1999]. Sections of Lefschetz fibrations play an important role in the theory. For example, in the presence of a section, the fundamental group and the signature of a Lefschetz fibration can be easily computed.

Here, we provide sections for genus 2 Lefschetz fibrations $\mathbb{C P}^{2} \# 13 \overline{\mathbb{C P}}^{2} \rightarrow$ $S^{2}$, with global monodromy given by the relation $\left(t_{c_{1}} t_{c_{2}} t_{c_{3}} t_{c_{4}} t_{c_{5}}^{2} t_{c_{4}} t_{c_{3}} t_{c_{2}} t_{c_{1}}\right)^{2}=1$ in the mapping class group $\Gamma_{2}$ of a closed genus 2 surface, where each $c_{i}$ is a simple closed curve as in Figure 3, and $t_{c_{i}}$ is a right-handed Dehn twist about $c_{i}$ for $i=1, \ldots, 5$. In [Korkmaz and Ozbagci 2008], similar relations were found in the mapping class group $\Gamma_{1, n}$ of a genus 1 surface with $n$ boundary components for $n=4, \ldots, 9$, giving an elliptic Lefschetz fibration $\mathbb{C P}^{2} \# 9 \overline{\mathbb{C P}}^{2} \rightarrow S^{2}$ with $n$ disjoint sections.

In Section 2, we recall definitions and relations in the mapping class group to be used in our computations, and we fix notation. In Section 3, we give brief background information on Lefschetz fibrations. In Section 4, we provide the necessary relations in the mapping class group $\Gamma_{2, n}$ for a genus 2 Lefschetz fibration $\mathbb{C P}^{2} \# 13 \overline{\mathbb{C P}}^{2} \rightarrow S^{2}$ with $n$ disjoint sections for $n=1, \ldots, 6$. In Section 5 we list several observations and open problems related to sections of Lefschetz fibrations. We show that a genus 2 Lefschetz fibration $\mathbb{C P}^{2} \# 13 \overline{\mathbb{C P}}^{2} \rightarrow S^{2}$ may admit at most 12 disjoint sections. We provide relations in the corresponding mapping class

MSC2000: primary 57M50; secondary 57R17.

Keywords: Lefschetz fibrations, sections of Lefschetz fibrations.

The author is supported in part by TUBITAK-2214. 
group that give $n=7$ and $n=8$ disjoint sections for genus 2 Lefschetz fibrations $\mathbb{C P} \mathbb{P}^{2} \# 13 \overline{\mathbb{C P}}^{2} \rightarrow S^{2}$. We conclude that any holomorphic genus 2 Lefschetz fibration without separating singular fibers admits a section.

\section{Mapping class groups}

Let $\Sigma_{g, n}^{k}$ denote an oriented, connected, genus $g$ surface, with $n$ boundary components and $k$ marked points. The mapping class group of $\Sigma_{g, n}^{k}$ is defined as the isotopy classes of orientation-preserving self-diffeomorphisms of $\Sigma_{g, n}^{k}$ that fix the marked points and the points on the boundary. Denote the mapping class group of $\Sigma_{g, n}^{k}$ by $\Gamma_{g, n}^{k}$. When $k=0$, denote the mapping class group of $\Sigma_{g, n}$ by $\Gamma_{g, n}$.

Let $a$ be a simple closed curve on $\Sigma_{g, n}^{k}$. A right-handed Dehn twist $t_{a}$ about $a$ is the isotopy class of a self-diffeomorphism of $\Sigma_{g, n}^{k}$ obtained by cutting $\Sigma_{g, n}^{k}$ along $a$ and gluing it back after twisting one side by $2 \pi$ to the right. The inverse of a right-handed Dehn twist is a left-handed Dehn twist, denoted by $t_{a}^{-1}$.

We now briefly mention the facts and relations to be used in our computations; for the proofs, see [Farb and Margalit 2005; Ivanov 2002]. If $f: \Sigma_{g, n}^{k} \rightarrow \Sigma_{g, n}^{k}$ is an orientation-preserving diffeomorphism, then $f t_{a} f^{-1}=t_{f(a)}$ for $a$ a simple closed curve on $\Sigma_{g, n}^{k}$.

For simplicity, we will denote a right-handed Dehn twist $t_{a}$ along $a$ by $a$, and a left-handed Dehn twist $t_{a}^{-1}$ by $\bar{a}$. The product $a b$ means that we first apply the Dehn twist $b$, then the Dehn twist $a$. A simple closed curve parallel to a boundary component of a given surface will be called a boundary curve of the surface.

The following relations will be useful:

The commutativity relation. If $a$ and $b$ are two disjoint simple closed curves on $\Sigma_{g, n}^{k}$, then the Dehn twists along $a$ and $b$ commute: $a b=b a$.

The braid relation. If $a$ and $b$ are two simple closed curves on $\Sigma_{g, n}^{k}$ intersecting transversely at a single point, then their Dehn twists satisfy $a b a=b a b$.

The lantern relation. Consider a sphere with four holes, the boundary curves $\delta_{1}, \delta_{2}, \delta_{3}, \delta_{4}$, and the simple closed curves $\alpha, \gamma, \sigma$, as shown in Figure 1 . We have $\delta_{1} \delta_{2} \delta_{3} \delta_{4}=\gamma \sigma \alpha$. Dehn discovered this relation; it was then rediscovered and named by D. Johnson.

The star relation [Gervais 2001]. Let $\Sigma_{1,3}$ be a torus with three boundary curves $\delta_{1}, \delta_{2}, \delta_{3}$. In $\Gamma_{1,3}$ we have $\delta_{1} \delta_{2} \delta_{3}=\left(a_{1} a_{2} a_{3} b\right)^{3}$ for the simple closed curves $a_{1}, a_{2}, a_{3}, b$ from Figure 1 .

The chain relation for a two-holed torus. Consider a torus $\Sigma_{1,2}$ with two boundary curves $\delta_{1}, \delta_{2}$, and the simple closed curves $c_{1}, c_{2}, b$, as shown in Figure 1. We have $\delta_{1} \delta_{2}=\left(c_{1} b c_{2}\right)^{4}$.

The chain relations for the genus 2 case: If $c_{1}, c_{2}, c_{3}, c_{4}, c_{5}$ is the chain of curves 


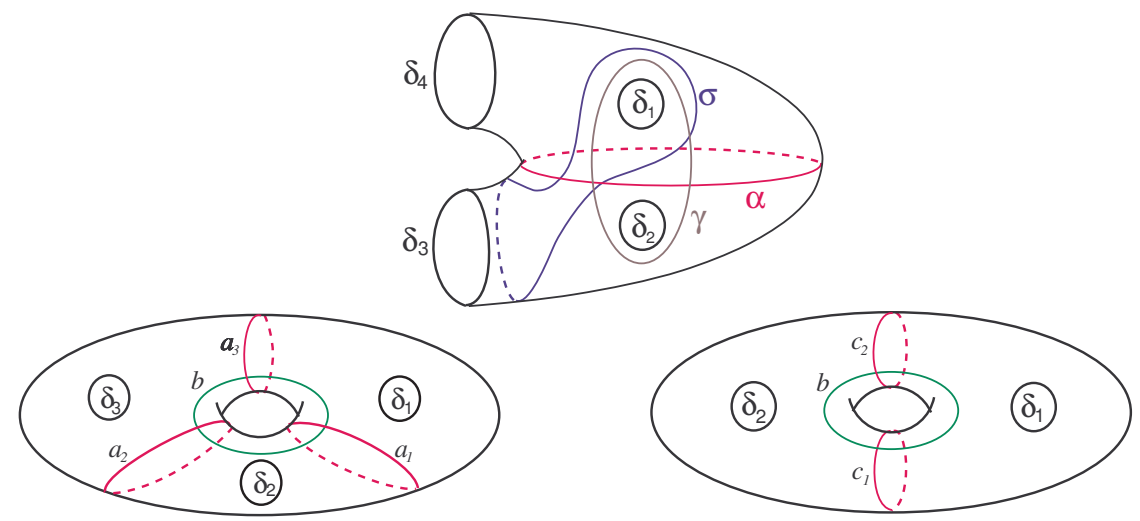

Figure 1. Counterclockwise from the top: The lantern relation, $\delta_{1} \delta_{2} \delta_{3} \delta_{4}=\gamma \sigma \alpha$, the star relation, $\delta_{1} \delta_{2} \delta_{3}=\left(a_{1} a_{2} a_{3} b\right)^{3}$, and the two-holed torus relation, $\delta_{1} \delta_{2}=\left(c_{1} b c_{2}\right)^{4}$.

shown in Figure 2, then for a genus 2 surface $\Sigma_{2,1}$ with one boundary curve $\delta_{1}$ we have $\delta_{1}=\left(c_{1} c_{2} c_{3} c_{4}\right)^{10}$, while for a genus 2 surface $\Sigma_{2,2}$ with two boundary curves $\delta_{1}, \delta_{2}$ we have $\delta_{1} \delta_{2}=\left(c_{1} c_{2} c_{3} c_{4} c_{5}\right)^{6}$.

\section{Lefschetz fibrations}

A Lefschetz fibration on a closed, connected, oriented smooth 4-manifold $X$ is a map $f: X \rightarrow \Sigma$, where $\Sigma$ is a closed, connected, oriented smooth surface, such that $f$ is surjective, has isolated critical points, and for each critical point $p$ there is an orientation-preserving local complex coordinate chart on which $f$ takes the form $f\left(z_{1}, z_{2}\right)=z_{1}^{2}+z_{2}^{2}$.

The Lefschetz fibration $f$ is a smooth fiber bundle away from critical points. A regular fiber of $f$ is diffeomorphic to a closed, oriented smooth genus $g$ surface. We define the genus of the Lefschetz fibration to be the genus of a regular fiber.

A singular fiber is a fiber containing a critical point. We assume that each singular fiber contains only one critical point. The singular fiber can be obtained by taking a simple closed curve on a regular fiber and shrinking it to a point. This
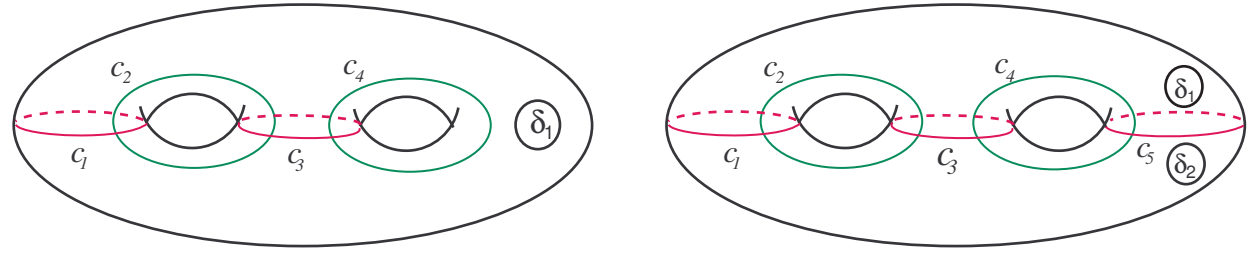

Figure 2. The chain relations: $\delta_{1}=\left(c_{1} c_{2} c_{3} c_{4}\right)^{10}$ and $\delta_{1} \delta_{2}=\left(c_{1} c_{2} c_{3} c_{4} c_{5}\right)^{6}$. 


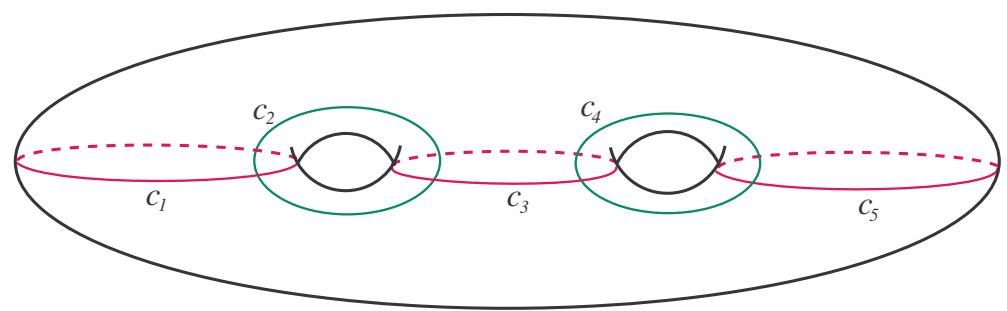

Figure 3. $\Sigma_{2}$.

simple closed curve describing the singular fiber is called a vanishing cycle. If this curve is a nonseparating curve, then the singular fiber is called nonseparating; otherwise it is called separating. For a genus $g$ Lefschetz fibration over $S^{2}$, the product of Dehn twists along the vanishing cycles gives us the global monodromy of the Lefschetz fibration.

On a closed surface $\Sigma_{g}$, the right-handed Dehn twists $c_{i}$ along the simple closed curves $c_{i}$ for $i=1, \ldots, s$, with the relation $c_{1} c_{2} \cdots c_{s}=1$, define a genus $g$ Lefschetz fibration over $S^{2}$ with vanishing cycles $c_{1}, \ldots, c_{s}$. In particular, in $\Gamma_{2}$ we have

$$
\begin{aligned}
& \left(c_{1} c_{2} c_{3} c_{4} c_{5}^{2} c_{4} c_{3} c_{2} c_{1}\right)^{2}=1, \\
& \left(c_{1} c_{2} c_{3} c_{4} c_{5}\right)^{6}=1, \\
& \left(c_{1} c_{2} c_{3} c_{4}\right)^{10}=1,
\end{aligned}
$$

where $c_{1}, \ldots, c_{5}$ are simple closed curves as in Figure 3. For each relation above, we have genus 2 Lefschetz fibrations over $S^{2}$ with total spaces $\mathbb{C P}^{2} \# 13 \overline{\mathbb{C P}}^{2}$, $\mathrm{K} 3 \# 2 \overline{\mathbb{C P}}^{2}$, and the Horikawa surface $H$, respectively. For more on Lefschetz fibrations, see [Auroux 2003; Gompf and Stipsicz 1999].

A section of a Lefschetz fibration is a map $\sigma: \Sigma \rightarrow X$ such that $f \sigma=\operatorname{id}_{\Sigma}$. Consider a collection of simple closed curves $c_{1}, \ldots, c_{s}$ on a genus $g$ surface $\Sigma_{g, n}$ with the relation

$$
c_{1} \cdots c_{s}=\delta_{1}^{k_{1}} \cdots \delta_{n}^{k_{n}}
$$

in $\Gamma_{g, n}$, where $\delta_{1}, \ldots, \delta_{n}$ are boundary curves, and $k_{1}, \ldots, k_{n}$ are positive integers. This relation defines a genus $g$ Lefschetz fibration over $S^{2}$ admitting $n$ disjoint sections, with global monodromy $c_{1} \cdots c_{s}=1$. Moreover, the self-intersection of the $i$-th section is $-k_{i}$. To see this, note that after gluing a disk along each boundary curve one gets the relation $c_{1} \cdots c_{s}=1$ in $\Gamma_{g}$. Thus, this relation will give us a genus $g$ Lefschetz fibration over $S^{2}$ as before. One can then use the centers of the capping disks to construct sections. For details, see [Gompf and Stipsicz 1999]; for more on self-intersection of sections, see [Smith 1998].

In the following sections, we will find relations of the above type, $c_{1} \cdots c_{s}=$ $\delta_{1} \cdots \delta_{n}$, in the mapping class group $\Gamma_{2, n}$ for $n=1, \ldots, 8$. 


\section{Relations in $\Gamma_{2, n}$}

For each $n=1, \ldots, 6$, we write the product of right-handed Dehn twists along the boundary curves $\delta_{1}, \ldots, \delta_{n}$ as a product of twenty right-handed Dehn twists along nonboundary parallel simple closed curves on a genus 2 surface $\Sigma_{2, n}$. Namely, we provide relations of the form $\delta_{1} \cdots \delta_{n}=\beta_{1} \cdots \beta_{20}$, where $\beta_{1}, \ldots, \beta_{20}$ are nonboundary parallel simple closed curves on $\Sigma_{2, n}$. After gluing disks along the boundary curves $\delta_{1}, \ldots, \delta_{n}$, we get the relation $1=\beta_{1} \cdots \beta_{20}$ in the mapping class group $\Gamma_{2}$. By using the commutativity relation and the braid relation, one can simplify the right-hand side of the equation $1=\beta_{1} \cdots \beta_{20}$ so that it gives us the global monodromy $\left(c_{1} c_{2} c_{3} c_{4} c_{5}^{2} c_{4} c_{3} c_{2} c_{1}\right)^{2}=1$ of a Lefschetz fibration $\mathbb{C P}^{2} \# 13 \overline{\mathbb{C P}}^{2} \rightarrow S^{2}$. For the simple closed curves $c_{1}, \ldots, c_{5}$, see Figure 3 . Here, twenty right-handed Dehn twists along nonboundary parallel, nonseparating simple closed curves $c_{i}$ correspond to twenty nonseparating singular fibers. The consecutive simple closed curves $c_{i}$ are the vanishing cycles.

In the following subsections, the relations we find in $\Gamma_{2, n}$ will give us a genus 2 Lefschetz fibration $\mathbb{C P}^{2} \# 13 \overline{\mathbb{C P}}^{2} \rightarrow S^{2}$ admitting $n$ disjoint sections of selfintersection -1 , for $n=1, \ldots, 6$.

4.1. Genus two surface with one hole. Consider the genus 2 surface $\Sigma_{2,1}$ with one boundary curve $\delta_{1}$, as in Figure 4 . We have

$$
\delta_{1}=\left(a_{1} b_{1} a_{2} b_{2}\right)^{10}=\left(a_{1} b_{1} a_{2} b_{2}\right)^{5}\left(a_{1} b_{1} a_{2} b_{2}\right)^{5} .
$$

Using the commutativity and braid relations, one can show

$$
\left(a_{1} b_{1} a_{2} b_{2}\right)^{5}=\left(a_{1} b_{1} a_{2}\right)^{4}\left(b_{2} a_{2} b_{1} a_{1}^{2} b_{1} a_{2} b_{2}\right) .
$$

(For the proof, see the appendix.) Notice the two-holed torus embedded in $\Sigma_{2,1}$ with two boundary curves $a_{3}, a_{4}$; then, by the chain relation for this torus, we have $\left(a_{1} b_{1} a_{2}\right)^{4}=a_{3} a_{4}$.

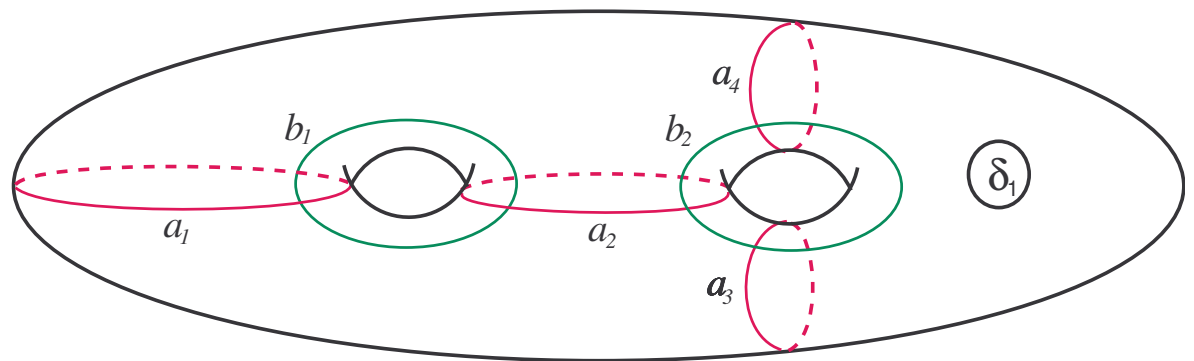

Figure 4. $\Sigma_{2,1}$. 


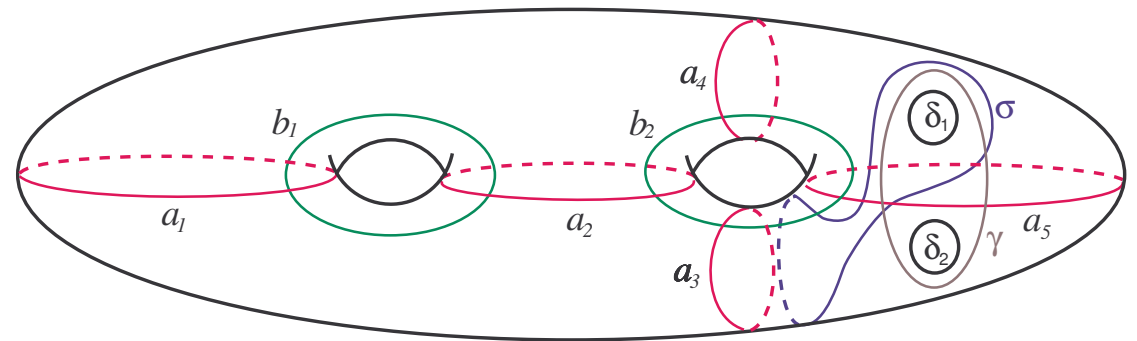

Figure 5. $\Sigma_{2,2}$.

By combining the relations above, we get

$$
\begin{aligned}
\delta_{1} & =\left(a_{1} b_{1} a_{2}\right)^{4}\left(b_{2} a_{2} b_{1} a_{1}^{2} b_{1} a_{2} b_{2}\right)\left(a_{1} b_{1} a_{2}\right)^{4}\left(b_{2} a_{2} b_{1} a_{1}^{2} b_{1} a_{2} b_{2}\right) \\
& =a_{3} a_{4}\left(b_{2} a_{2} b_{1} a_{1}^{2} b_{1} a_{2} b_{2}\right) a_{3} a_{4}\left(b_{2} a_{2} b_{1} a_{1}^{2} b_{1} a_{2} b_{2}\right) \\
& =\left(a_{3} a_{4} b_{2} a_{2} b_{1} a_{1}^{2} b_{1} a_{2} b_{2}\right)^{2} .
\end{aligned}
$$

4.2. Genus two surface with two holes. Consider the genus 2 surface $\Sigma_{2,2}$ with two boundary curves $\delta_{1}, \delta_{2}$ from Figure 5, and notice the embedded sphere embedded in $\Sigma_{2,2}$ with four boundary curves $\delta_{1}, \delta_{2}, a_{3}, a_{4}$. Then, using the lantern relation, we have

$$
a_{3} a_{4} \delta_{1} \delta_{2}=\gamma \sigma a_{5} .
$$

Notice in Figure 5 the genus 2 surface with one boundary curve $\gamma$; we thus have the chain relation $\gamma=\left(a_{1} b_{1} a_{2} b_{2}\right)^{10}$. Substituting $\gamma$ in the lantern relation and then using the two-holed torus relation $\left(a_{1} b_{1} a_{2}\right)^{4}=a_{3} a_{4}$ in the equation, we get

$$
\begin{aligned}
a_{3} a_{4} \delta_{1} \delta_{2} & =\gamma \sigma a_{5} \\
& =\left(a_{1} b_{1} a_{2} b_{2}\right)^{10} \sigma a_{5} \\
& =\left(a_{1} b_{1} a_{2} b_{2}\right)^{5}\left(a_{1} b_{1} a_{2} b_{2}\right)^{5} \sigma a_{5} \\
& =\left(a_{1} b_{1} a_{2}\right)^{4}\left(b_{2} a_{2} b_{1} a_{1}^{2} b_{1} a_{2} b_{2}\right)\left(a_{1} b_{1} a_{2}\right)^{4}\left(b_{2} a_{2} b_{1} a_{1}^{2} b_{1} a_{2} b_{2}\right) \sigma a_{5} \\
& =a_{3} a_{4}\left(b_{2} a_{2} b_{1} a_{1}^{2} b_{1} a_{2} b_{2}\right) a_{3} a_{4}\left(b_{2} a_{2} b_{1} a_{1}^{2} b_{1} a_{2} b_{2}\right) \sigma a_{5} .
\end{aligned}
$$

We simplify this equation to

$$
\delta_{1} \delta_{2}=b_{2} a_{2} b_{1} a_{1}^{2} b_{1} a_{2} b_{2} a_{3} a_{4}\left(b_{2} a_{2} b_{1} a_{1}^{2} b_{1} a_{2} b_{2}\right) \sigma a_{5} .
$$

4.3. Genus two surface with three holes. First, the lantern relation for the sphere with four boundary curves $\delta_{1}, \delta_{2}, a_{4}, a_{5}$ in $\Sigma_{2,3}$ from Figure 6 is $a_{4} a_{5} \delta_{1} \delta_{2}=\gamma \sigma a_{6}$. For the three-holed torus with boundary curves $\gamma, a_{1}, a_{2}$, we have the star relation $\gamma a_{1} a_{2}=\left(a_{4} a_{5} a_{3} b_{2}\right)^{3}$, while for the three-holed torus with boundary curves $\delta_{3}, a_{4}, a_{5}$, we have $\delta_{3} a_{4} a_{5}=\left(a_{1} a_{2} a_{3} b_{1}\right)^{3}$. 


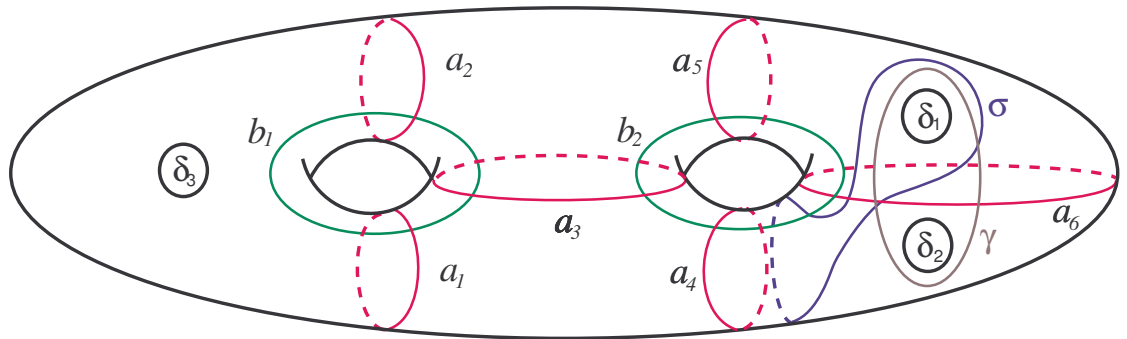

Figure 6. $\Sigma_{2,3}$.

Now, combine these relations, substitute $\delta_{3}$ and $\gamma$, then simplify by using the commutativity and braid relations. Note that all the $a_{i}$ commute for $i=1, \ldots, 6$. The simple closed curves $a_{1}, a_{2}, a_{3}$ intersect $b_{1}$ transversely at a single point, and $a_{4}, a_{5}, a_{3}$ intersect $b_{2}$ transversely at a single point. Thus, with $\beta=\bar{a}_{5} \bar{a}_{4} b_{2} a_{4} a_{5}$,

$$
\begin{aligned}
\delta_{1} \delta_{2} & =\bar{a}_{4} \bar{a}_{5} \gamma \sigma a_{6}, \\
\delta_{1} \delta_{2} \delta_{3} & =\delta_{3} \bar{a}_{4} \bar{a}_{5} \gamma \sigma a_{6} \\
& =\delta_{3} \bar{a}_{4} \bar{a}_{5} \bar{a}_{1} \bar{a}_{2}\left(a_{4} a_{5} a_{3} b_{2}\right)^{3} \sigma a_{6} \\
& =\bar{a}_{1} \bar{a}_{2}\left(\delta_{3}\right) \bar{a}_{4} \bar{a}_{5}\left(a_{4} a_{5} a_{3} b_{2}\right)\left(a_{4} a_{5} a_{3} b_{2}\right)^{2} \sigma a_{6} \\
& =\bar{a}_{1} \bar{a}_{2}\left(\left(a_{1} a_{2} a_{3} b_{1}\right)^{3} \bar{a}_{4} \bar{a}_{5}\right) a_{3} b_{2}\left(a_{4} a_{5} a_{3} b_{2}\right)^{2} \sigma a_{6} \\
& =a_{3} b_{1}\left(a_{1} a_{2} a_{3} b_{1}\right)^{2} a_{3}\left(\bar{a}_{4} \bar{a}_{5} b_{2} a_{4} a_{5}\right) a_{3} b_{2} a_{4} a_{5} a_{3} b_{2} \sigma a_{6} \\
& =a_{3} b_{1}\left(a_{1} a_{2} a_{3} b_{1}\right)^{2} a_{3} \beta a_{3} b_{2} a_{4} a_{5} a_{3} b_{2} \sigma a_{6} .
\end{aligned}
$$

4.4. Genus two surface with four holes. We will use the three-holed genus two relation we found in Section 4.3. Notice in Figure 7 the genus 2 surface with three boundary curves $\delta_{3}, \delta_{4}, \gamma$. Then

$$
\begin{aligned}
\delta_{3} \delta_{4} \gamma & =a_{3} b_{2}\left(a_{5} a_{4} a_{3} b_{2}\right)^{2} a_{3} \beta_{1} a_{3} b_{1} a_{2} a_{1} a_{3} b_{1} \sigma_{1} a_{7} \\
& =a_{3} \beta_{1} a_{3} b_{1} a_{2} a_{1} a_{3} b_{1} \sigma_{1} a_{7} a_{3} b_{2}\left(a_{5} a_{4} a_{3} b_{2}\right)^{2} .
\end{aligned}
$$

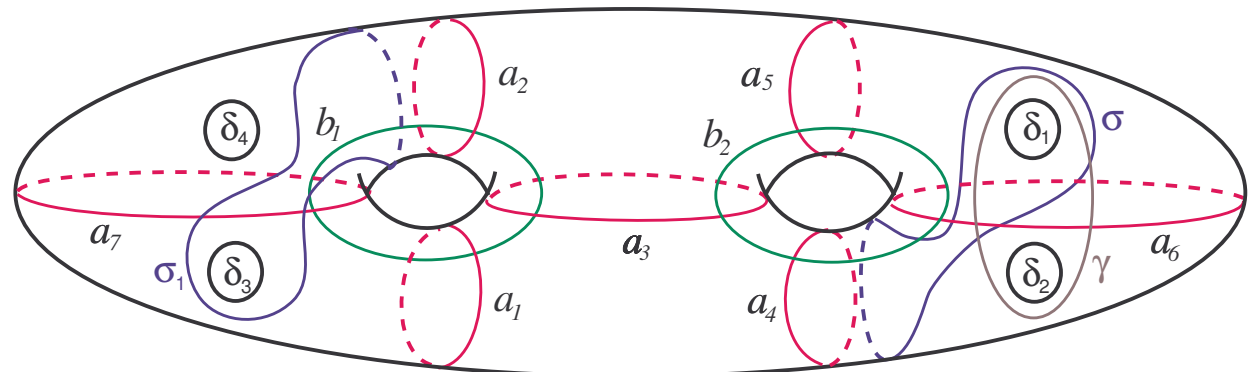

Figure 7. $\Sigma_{2,4}$. 
where $\beta_{1}=\bar{a}_{1} \bar{a}_{2} b_{1} a_{2} a_{1}$. Note that we identify the curves $\left(a_{1}, a_{2}, a_{3}, a_{4}, a_{5}, a_{6}\right)$ in $\Sigma_{2,3}$ from Figure 6 with the curves $\left(a_{5}, a_{4}, a_{3}, a_{2}, a_{1}, a_{7}\right)$ in $\Sigma_{2,4}$ from Figure 7.

By the lantern relation for the sphere with four boundary curves $\delta_{1}, \delta_{2}, a_{4}, a_{5}$, we have

$$
a_{4} a_{5} \delta_{1} \delta_{2}=\gamma \sigma a_{6} .
$$

Now, combine the above relations to get

$$
\begin{aligned}
\delta_{1} \delta_{2} \delta_{3} \delta_{4} & =a_{3} \beta_{1} a_{3} b_{1} a_{2} a_{1} a_{3} b_{1} \sigma_{1} a_{7} a_{3} b_{2}\left(a_{5} a_{4} a_{3} b_{2}\right)^{2} \bar{\gamma} \bar{a}_{4} \bar{a}_{5} \gamma \sigma a_{6} \\
& =a_{3} \beta_{1} a_{3} b_{1} a_{2} a_{1} a_{3} b_{1} \sigma_{1} a_{7} a_{3} b_{2}\left(a_{5} a_{4} a_{3} b_{2}\right) a_{3} a_{5} a_{4} b_{2}\left(\bar{a}_{4} \bar{a}_{5} \sigma a_{6}\right) \\
& =a_{3} \beta_{1} a_{3} b_{1} a_{2} a_{1} a_{3} b_{1} \sigma_{1} a_{7} a_{3} b_{2}\left(a_{5} a_{4} a_{3} b_{2}\right) a_{3} \beta_{2} \sigma a_{6},
\end{aligned}
$$

where $\beta_{1}=\bar{a}_{1} \bar{a}_{2} b_{1} a_{2} a_{1}$ and $\beta_{2}=a_{5} a_{4} b_{2} \bar{a}_{4} \bar{a}_{5}$.

4.5. Genus two surface with five holes. The lantern relation for the sphere with four boundary curves $\delta_{1}, \delta_{2}, a_{4}, a_{5}$ in $\Sigma_{2,5}$ from Figure 8 is $a_{4} a_{5} \delta_{1} \delta_{2}=\gamma \sigma a_{6}$. In Figure 8 , notice the genus 2 surface with four boundary curves $\delta_{3}, \delta_{4}, \delta_{5}, \gamma$. Identify the curves $\left(\delta_{1}, \delta_{2}, a_{5}, a_{6}, \sigma\right)$ in $\Sigma_{2,4}$ from Figure 7 with $\left(\delta_{5}, \gamma, a_{8}, a_{5}, \sigma_{2}\right)$ in $\Sigma_{2,5}$ from Figure 8. Then, by the relation given in Section 4.4, we have

$$
\delta_{3} \delta_{4} \delta_{5} \gamma=a_{3} \beta_{1} a_{3} b_{1} a_{2} a_{1} a_{3} b_{1} \sigma_{1} a_{7} a_{3} b_{2}\left(a_{8} a_{4} a_{3} b_{2}\right) a_{3} \beta_{2} \sigma_{2} a_{5},
$$

where $\beta_{1}=\bar{a}_{1} \bar{a}_{2} b_{1} a_{2} a_{1}$ and $\beta_{2}=a_{8} a_{4} b_{2} \bar{a}_{4} \bar{a}_{8}$.

Now, combine the above relations and simplify the equation as

$$
\begin{aligned}
\delta_{1} \delta_{2} \delta_{3} \delta_{4} \delta_{5} & =a_{3} \beta_{1} a_{3} b_{1} a_{2} a_{1} a_{3} b_{1} \sigma_{1} a_{7} a_{3} b_{2}\left(a_{8} a_{4} a_{3} b_{2}\right) a_{3} \beta_{2} \sigma_{2} a_{5} \bar{\gamma} \bar{a}_{4} \bar{a}_{5} \gamma \sigma a_{6} \\
& =a_{3} \beta_{1} a_{3} b_{1} a_{2} a_{1} a_{3} b_{1} \sigma_{1} a_{7} a_{3} b_{2}\left(a_{8} a_{4} a_{3} b_{2}\right) a_{3} \beta_{2} \sigma_{2} \bar{a}_{4} \sigma a_{6} \\
& =a_{3} \beta_{1} a_{3} b_{1} a_{2} a_{1} a_{3} b_{1} \sigma_{1} a_{7} a_{3}\left(\bar{a}_{4}\right) b_{2}\left(a_{4} a_{8} a_{3} b_{2}\right) a_{3} \beta_{2} \sigma_{2} \sigma a_{6} \\
& =a_{3} \beta_{1} a_{3} b_{1} a_{2} a_{1} a_{3} b_{1} \sigma_{1} a_{7} a_{3} \beta_{3}\left(a_{8} a_{3} b_{2}\right) a_{3} \beta_{2} \sigma_{2} \sigma a_{6},
\end{aligned}
$$

where $\beta_{1}=\bar{a}_{1} \bar{a}_{2} b_{1} a_{1} a_{2}, \beta_{2}=a_{8} a_{4} b_{2} \bar{a}_{4} \bar{a}_{8}$, and $\beta_{3}=\bar{a}_{4} b_{2} a_{4}$.

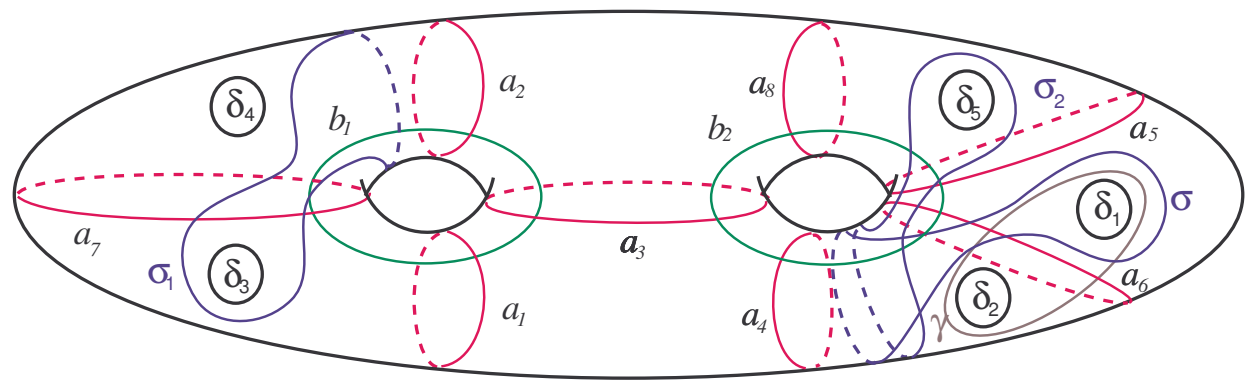

Figure 8. $\Sigma_{2,5}$. 


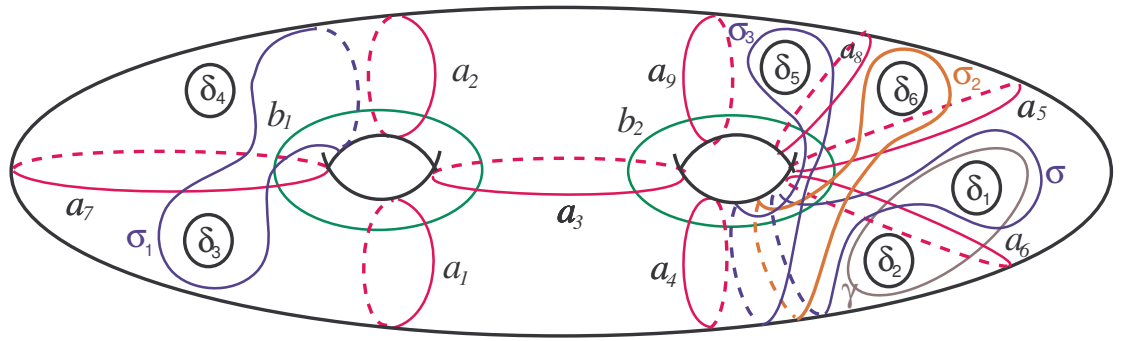

Figure 9. $\Sigma_{2,6}$.

4.6. Genus two surface with six holes. The lantern relation for the sphere with four boundary curves $\delta_{1}, \delta_{2}, a_{4}, a_{5}$ in $\Sigma_{2,6}$ from Figure 9 is $a_{4} a_{5} \delta_{1} \delta_{2}=\gamma \sigma a_{6}$. Now, identify the curves $\left(\delta_{1}, \delta_{2}, a_{6}, a_{8}, \sigma, \sigma_{2}\right)$ in $\Sigma_{2,5}$ from Figure 8 with the curves $\left(\delta_{6}, \gamma, a_{5}, a_{9}, \sigma_{2}, \sigma_{3}\right)$ in $\Sigma_{2,6}$ from Figure 9. By the relation given in Section 4.5 for the genus 2 surface with five boundary curves $\delta_{3}, \delta_{4}, \delta_{5}, \delta_{6}, \gamma$, we have

$$
\delta_{6} \gamma \delta_{3} \delta_{4} \delta_{5}=a_{3} \beta_{1} a_{3} b_{1} a_{2} a_{1} a_{3} b_{1} \sigma_{1} a_{7} a_{3} \beta_{3} a_{9} a_{3} b_{2} a_{3} \beta_{2} \sigma_{3} \sigma_{2} a_{5} .
$$

where $\beta_{1}=\bar{a}_{1} \bar{a}_{2} b_{1} a_{2} a_{1}, \beta_{2}=a_{9} a_{4} b_{2} \bar{a}_{4} \bar{a}_{9}$, and $\beta_{3}=\bar{a}_{4} b_{2} a_{4}$.

Now, combine the above relations to get

$$
\begin{aligned}
\delta_{1} \delta_{2} \delta_{3} \delta_{4} \delta_{5} \delta_{6} & =a_{3} \beta_{1} a_{3} b_{1} a_{2} a_{1} a_{3} b_{1} \sigma_{1} a_{7} a_{3} \beta_{3} a_{9} a_{3} b_{2} a_{3} \beta_{2} \sigma_{3} \sigma_{2} a_{5} \bar{\gamma} \bar{a}_{4} \bar{a}_{5} \gamma \sigma a_{6} \\
& =a_{3} \beta_{1} a_{3} b_{1} a_{2} a_{1} a_{3} b_{1} \sigma_{1} a_{7} a_{3} \beta_{3} a_{9} a_{3} b_{2} a_{3} \beta_{2} \sigma_{3} \sigma_{2}\left(\bar{a}_{4}\right) \sigma a_{6} \\
& =a_{3} \beta_{1} a_{3} b_{1} a_{2} a_{1} a_{3} b_{1} \sigma_{1} a_{7} a_{3}\left(\bar{a}_{4}\right)\left(\beta_{3}\right) a_{9} a_{3} b_{2} a_{3} \beta_{2} \sigma_{3} \sigma_{2} \sigma a_{6} \\
& =a_{3} \beta_{1} a_{3} b_{1} a_{2} a_{1} a_{3} b_{1} \sigma_{1} a_{7} a_{3}\left(\bar{a}_{4}\right)\left(\bar{a}_{4} b_{2} a_{4}\right) a_{9}\left(a_{3} b_{2} a_{3}\right) \beta_{2} \sigma_{3} \sigma_{2} \sigma a_{6} \\
& =a_{3} \beta_{1} a_{3} b_{1} a_{2} a_{1} a_{3} b_{1} \sigma_{1} a_{7} a_{3}\left(\bar{a}_{4}\right)\left(b_{2} a_{4} \bar{b}_{2}\right) a_{9}\left(b_{2} a_{3} b_{2}\right) \beta_{2} \sigma_{3} \sigma_{2} \sigma a_{6} \\
& =a_{3} \beta_{1} a_{3} b_{1} a_{2} a_{1} a_{3} b_{1} \sigma_{1} a_{7} a_{3} \beta_{3} \beta_{4} a_{3} b_{2} \beta_{2} \sigma_{3} \sigma_{2} \sigma a_{6}
\end{aligned}
$$

where $\beta_{1}=\bar{a}_{1} \bar{a}_{2} b_{1} a_{2} a_{1}, \beta_{2}=a_{5} a_{4} b_{2} \bar{a}_{4} \bar{a}_{5}, \beta_{3}=\bar{a}_{4} b_{2} a_{4}=b_{2} a_{4} \bar{b}_{2}$, and $\beta_{4}=\bar{b}_{2} a_{9} b_{2}$.

\section{Final remarks}

Lemma 5.1. A genus 2 Lefschetz fibration $\mathbb{C P}^{2} \# 13 \overline{\mathbb{C P}}^{2} \rightarrow S^{2}$ admits at most 12 disjoint sections.

Proof. Suppose that $\mathbb{C P}^{2} \# 13 \overline{\mathbb{C P}}^{2} \rightarrow S^{2}$ admits 13 disjoint sections. Each section is a sphere with self-intersection -1 . Furthermore, each section intersects a regular fiber, a genus 2 surface $\Sigma_{2}$ with self-intersection 0 , at one point. Now, by blowing down all -1 spheres, we get a genus 2 surface $\widetilde{\Sigma}_{2}$ with self-intersection 13 , which cannot exist in a manifold with second homology $\mathbb{Z}$. 
In Section 4, we found relations giving $n$ disjoint sections for genus 2 Lefschetz fibration $\mathbb{C P}^{2} \# 13 \overline{\mathbb{C P}}^{2} \rightarrow S^{2}$ for $n=1, \ldots, 6$. The technique applied in Section 4 stops at $n=6$. However, by using results from [Korkmaz and Ozbagci 2008], we can find relations in the corresponding mapping class group that give $n=7$ and $n=8$ disjoint sections for genus 2 Lefschetz fibration $\mathbb{C P}^{2} \# 13 \overline{\mathbb{C P}}^{2} \rightarrow S^{2}$. We will next show how to derive these relations. This method does not go further, and it remains unknown whether there are more than eight sections.

The seven-holed torus relation from [Korkmaz and Ozbagci 2008] sits in $\Sigma_{2,7}$ :

$$
\delta_{1} \delta_{2} \delta_{3} \delta_{4} \delta_{5} \delta_{6} \delta_{7}=\alpha_{3} \alpha_{4} \alpha_{1} b_{1} \sigma_{5} \alpha_{2} \beta_{5} \sigma_{3} \sigma_{6} \alpha_{6} \beta_{3} \sigma_{4},
$$

where $\beta_{3}=\alpha_{3} b_{1} \bar{\alpha}_{3}$ and $\beta_{5}=\alpha_{5} b_{1} \bar{\alpha}_{5}$ in $\Sigma_{2,7}$; see Figure 10 . We identify the boundary curves $\left(\delta_{1}, \delta_{2}, \delta_{3}, \delta_{4}, \delta_{5}, \delta_{6}, \delta_{7}\right)$ in $\Sigma_{1,7}$ from Figure 10 with the curves $\left(\delta_{6}, \delta_{5}, a_{2}, a_{1}, \delta_{2}, \delta_{1}, \delta_{7}\right)$ in $\Sigma_{2,7}$. The seven-holed torus relation gives

$$
a_{1} a_{2} \delta_{1} \delta_{2} \delta_{5} \delta_{6} \delta_{7}=a_{3} a_{4} a_{9} b_{2} \sigma_{5} a_{10} \beta_{5} \sigma_{3} \sigma_{6} a_{5} \beta_{3} \sigma_{4}
$$

where $\beta_{3}=a_{3} b_{2} \bar{a}_{3}$ and $\beta_{5}=a_{6} b_{2} \bar{a}_{6}$.

Next, we combine this with the lantern relation $a_{1} a_{2} \delta_{3} \delta_{4}=\gamma \sigma a_{7}$ for the sphere with four boundary curves $\delta_{3}, \delta_{4}, a_{1}, a_{2}$ in $\Sigma_{2,7}$. The star relation for the torus with three boundary curves $\gamma, a_{4}, a_{10}$ is $a_{4} a_{10} \gamma=\left(a_{1} a_{2} a_{3} b_{1}\right)^{3}$. We now substitute
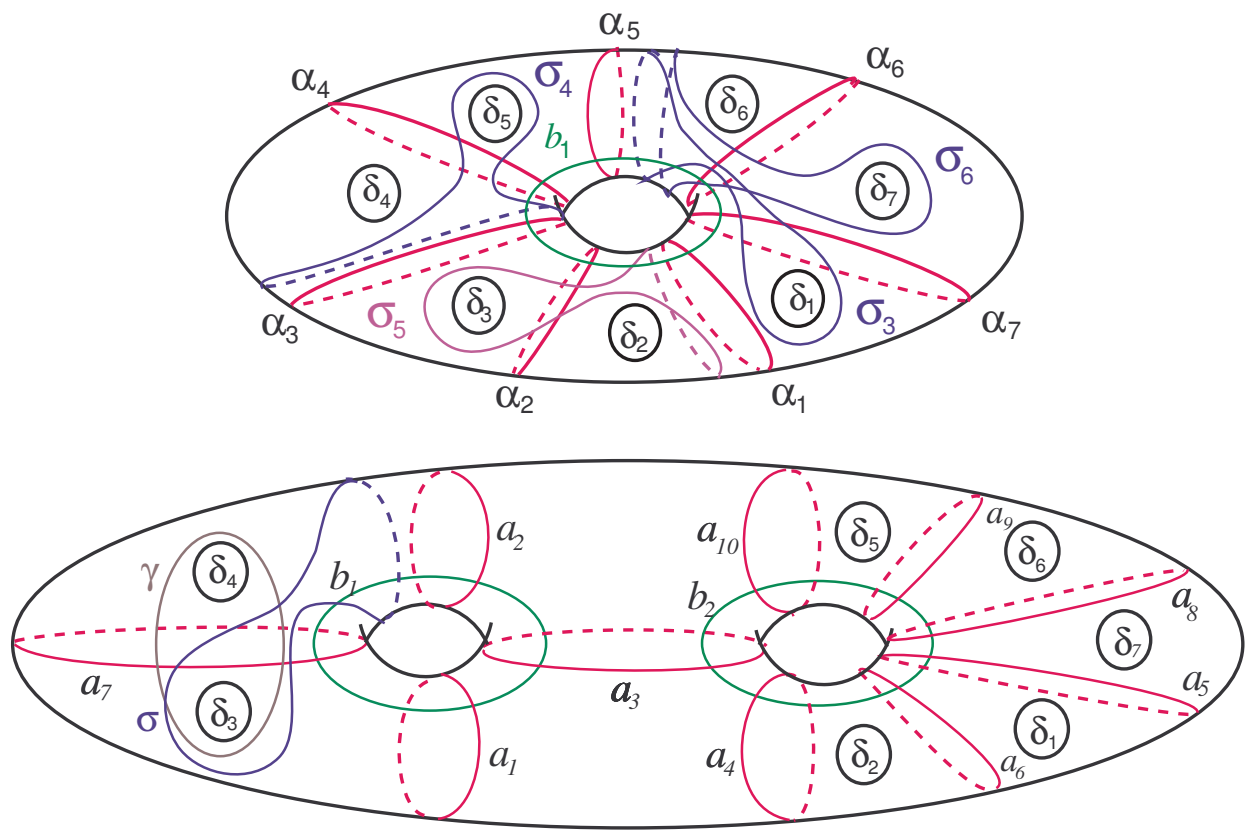

Figure 10. Seven-holed torus relation. 

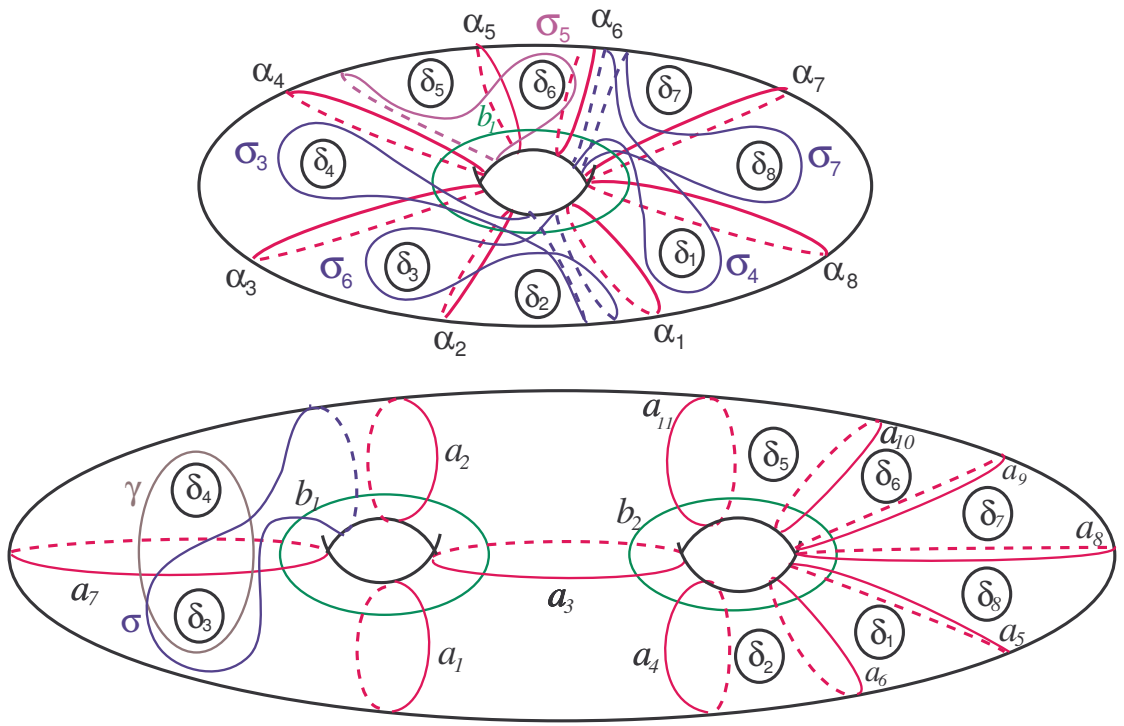

Figure 11. Eight-holed torus relation.

$\gamma=\bar{a}_{4} \bar{a}_{10}\left(a_{1} a_{2} a_{3} b_{1}\right)^{3}$ into the lantern relation; then we simplify the equation and write the product of right-handed Dehn twists along the boundary curves $\delta_{1}, \ldots, \delta_{7}$ as a product of twenty right-handed Dehn twists along nonboundary parallel simple closed curves on $\Sigma_{2,7}$ :

$\delta_{1} \delta_{2} \delta_{3} \delta_{4} \delta_{5} \delta_{6} \delta_{7}$

$$
\begin{aligned}
& =a_{3} a_{4} a_{9} b_{2} \sigma_{5} a_{10} \beta_{5} \sigma_{3} \sigma_{6} a_{5} \beta_{3} \sigma_{4} \bar{a}_{1} \bar{a}_{2} \bar{a}_{1} \bar{a}_{2} \gamma \sigma a_{7} \\
& =a_{3} a_{9} b_{2} \sigma_{5} a_{10} \beta_{5} \sigma_{3} \sigma_{6} a_{5} \beta_{3} \sigma_{4}\left(a_{4}\right) \bar{a}_{1} \bar{a}_{2} \bar{a}_{1} \bar{a}_{2} \bar{a}_{4} \bar{a}_{10}\left(a_{1} a_{2} a_{3} b_{1}\right)^{3} \sigma a_{7} \\
& =a_{3} a_{9} b_{2} \sigma_{5} a_{10} \beta_{5} \sigma_{3} \sigma_{6} a_{5} \beta_{3} \sigma_{4} \bar{a}_{1} \bar{a}_{2} a_{3} b_{1}\left(a_{1} a_{2} a_{3} b_{1}\right)^{2}\left(\bar{a}_{10}\right) \sigma a_{7} \\
& =a_{3} a_{9}\left(\bar{a}_{10}\right) b_{2} \sigma_{5} a_{10} \beta_{5} \sigma_{3} \sigma_{6} a_{5} \beta_{3} \sigma_{4} a_{3}\left(\bar{a}_{1} \bar{a}_{2} b_{1} a_{1} a_{2}\right) a_{3} b_{1}\left(a_{1} a_{2} a_{3} b_{1}\right) \sigma a_{7} \\
& =a_{3} a_{9}\left(\bar{a}_{10} b_{2} a_{10}\right)\left(\bar{a}_{10} \sigma_{5} a_{10}\right) \beta_{5} \sigma_{3} \sigma_{6} a_{5} \beta_{3} \sigma_{4} a_{3} \tilde{\beta} a_{3} b_{1}\left(a_{1} a_{2} a_{3} b_{1}\right) \sigma a_{7} \\
& =a_{3} a_{9} \tilde{\beta}_{1} \tilde{\beta}_{2} \beta_{5} \sigma_{3} \sigma_{6} a_{5} \beta_{3} \sigma_{4} a_{3} \tilde{\beta} a_{3} b_{1}\left(a_{1} a_{2} a_{3} b_{1}\right) \sigma a_{7},
\end{aligned}
$$

where $\tilde{\beta}_{1}=\bar{a}_{10} b_{2} a_{10}, \quad \tilde{\beta}_{2}=\bar{a}_{10} \sigma_{5} a_{10}, \quad \beta_{5}=a_{6} b_{2} \bar{a}_{6}, \quad \beta_{3}=a_{3} b_{2} \bar{a}_{3}$, and $\tilde{\beta}=$ $\bar{a}_{1} \bar{a}_{2} b_{1} a_{1} a_{2}$. Note that the simple closed curves $\sigma_{5}$ and $a_{10}$ intersect at 2 points.

Similarly, the eight-holed torus relation from [Korkmaz and Ozbagci 2008] sits in $\Sigma_{2,8}$ (see Figure 11):

$\delta_{1} \delta_{2} \delta_{3} \delta_{4} \delta_{5} \delta_{6} \delta_{7} \delta_{8}=\alpha_{4} \alpha_{5} \beta_{1} \sigma_{3} \sigma_{6} \alpha_{2} \beta_{6} \sigma_{4} \sigma_{7} \alpha_{7} \beta_{4} \sigma_{5}=\alpha_{4} b_{1} \sigma_{5} \alpha_{5} \beta_{1} \sigma_{3} \sigma_{6} \alpha_{2} \beta_{6} \sigma_{4} \sigma_{7} \alpha_{7}$, where $\beta_{1}=\alpha_{1} b_{1} \bar{\alpha}_{1}, \beta_{4}=\alpha_{4} b_{1} \bar{\alpha}_{4}$, and $\beta_{6}=\alpha_{6} b_{1} \bar{\alpha}_{6}$ in $\Sigma_{2,8}$. We identify the curves 
$\left(\delta_{1}, \delta_{2}, \delta_{3}, \delta_{4}, \delta_{5}, \delta_{6}, \delta_{7}, \delta_{8}\right)$ in $\Sigma_{1,8}$ with $\left(\delta_{1}, \delta_{8}, \delta_{7}, \delta_{6}, \delta_{5}, a_{2}, a_{1}, \delta_{2}\right)$ in $\Sigma_{2,8}$.

By applying the same technique, we also get the necessary relation for $n=8$. The eight-holed torus relation gives

$$
a_{1} a_{2} \delta_{1} \delta_{2} \delta_{5} \delta_{6} \delta_{7} \delta_{8}=a_{10} b_{2} \sigma_{5} a_{11} \beta_{1} \sigma_{3} \sigma_{6} a_{8} \beta_{6} \sigma_{4} \sigma_{7} a_{4},
$$

where $\beta_{1}=a_{5} b_{2} \bar{a}_{5}$ and $\beta_{6}=a_{3} b_{2} \bar{a}_{3}$. We combine this with the lantern relation $a_{1} a_{2} \delta_{3} \delta_{4}=\gamma \sigma a_{7}$ for the sphere with four boundary curves $\delta_{3}, \delta_{4}, a_{1}, a_{2}$ in $\Sigma_{2,8}$. Using the star relation $a_{4} a_{11} \gamma=\left(a_{1} a_{2} a_{3} b_{1}\right)^{3}$ for the torus with three boundary curves $\gamma, a_{4}, a_{11}$, we substitute $\gamma=\bar{a}_{4} \bar{a}_{11}\left(a_{1} a_{2} a_{3} b_{1}\right)^{3}$ in the lantern relation. We simplify the equation as

$$
\begin{aligned}
\delta_{1} \delta_{2} \delta_{3} \delta_{4} \delta_{5} \delta_{6} \delta_{7} \delta_{8} \\
=a_{10} b_{2} \sigma_{5} a_{11} \beta_{1} \sigma_{3} \sigma_{6} a_{8} \beta_{6} \sigma_{4} \sigma_{7} a_{4} \bar{a}_{1} \bar{a}_{2} \bar{a}_{1} \bar{a}_{2} \gamma \sigma a_{7} \\
=a_{10} b_{2} \sigma_{5} a_{11} \beta_{1} \sigma_{3} \sigma_{6} a_{8} \beta_{6} \sigma_{4} \sigma_{7} a_{4} \bar{a}_{1} \bar{a}_{2} \bar{a}_{1} \bar{a}_{2} \bar{a}_{4} \bar{a}_{11}\left(a_{1} a_{2} a_{3} b_{1}\right)^{3} \sigma a_{7} \\
=a_{10} b_{2} \sigma_{5} a_{11} \beta_{1} \sigma_{3} \sigma_{6} a_{8} \beta_{6} \sigma_{4} \sigma_{7} \bar{a}_{1} \bar{a}_{2} a_{3} b_{1}\left(a_{1} a_{2} a_{3} b_{1}\right)^{2}\left(\bar{a}_{11}\right) \sigma a_{7} \\
=a_{10}\left(\bar{a}_{11}\right) b_{2} \sigma_{5} a_{11} \beta_{1} \sigma_{3} \sigma_{6} a_{8} \beta_{6} \sigma_{4} \sigma_{7} a_{3}\left(\bar{a}_{1} \bar{a}_{2} b_{1} a_{1} a_{2}\right) a_{3} b_{1}\left(a_{1} a_{2} a_{3} b_{1}\right) \sigma a_{7} \\
=a_{10}\left(\bar{a}_{11} b_{2} a_{11}\right)\left(\bar{a}_{11} \sigma_{5} a_{11}\right) \beta_{1} \sigma_{3} \sigma_{6} a_{8} \beta_{6} \sigma_{4} \sigma_{7} a_{3} \tilde{\beta} a_{3} b_{1}\left(a_{1} a_{2} a_{3} b_{1}\right) \sigma a_{7} \\
=a_{10} \tilde{\beta}_{1} \tilde{\beta}_{2} \beta_{1} \sigma_{3} \sigma_{6} a_{8} \beta_{6} \sigma_{4} \sigma_{7} a_{3} \tilde{\beta} a_{3} b_{1}\left(a_{1} a_{2} a_{3} b_{1}\right) \sigma a_{7},
\end{aligned}
$$

where $\beta_{1}=a_{5} b_{2} \bar{a}_{5}, \quad \beta_{6}=a_{3} b_{2} \bar{a}_{3}, \tilde{\beta}=\bar{a}_{1} \bar{a}_{2} b_{1} a_{1} a_{2}, \quad \tilde{\beta}_{1}=\bar{a}_{11} \beta_{2} a_{11}$ and $\tilde{\beta}_{2}=$ $\bar{a}_{11} \sigma_{5} a_{11}$. Note that the simple closed curves $\sigma_{5}$ and $a_{11}$ intersect at 2 points.

By Lemma 5.1, for $n>12$ there is no relation in the mapping class group $\Gamma_{2, n}$ inducing a genus 2 Lefschetz fibration $\mathbb{C P}^{2} \# 13 \overline{\mathbb{C P}}^{2} \rightarrow S^{2}$ with $n$ disjoint sections. For $n=1, \ldots, 8$, we did find relations giving $n$ disjoint sections for genus 2 Lefschetz fibration $\mathbb{C P}^{2} \# 13 \overline{\mathbb{C P}}^{2} \rightarrow S^{2}$. As a consequence, by using a result of K. Chakiris, we observe:

Corollary 5.2. Any genus 2 holomorphic Lefschetz fibration without separating singular fibers admits a section.

Proof. Chakiris [1978] showed that any genus 2 holomorphic Lefschetz fibration without separating singular fibers is obtained by fiber-summing the three genus 2 Lefschetz fibrations given by the relations

$$
\left(c_{1} c_{2} c_{3} c_{4} c_{5}^{2} c_{4} c_{3} c_{2} c_{1}\right)^{2}=1, \quad\left(c_{1} c_{2} c_{3} c_{4} c_{5}\right)^{6}=1, \quad \text { and } \quad\left(c_{1} c_{2} c_{3} c_{4}\right)^{10}=1
$$

in $\Gamma_{2}$, where $c_{1}, \ldots, c_{5}$ are the simple closed curves shown in Figure 3. As noted in Section 2, each relation gives us a genus 2 Lefschetz fibration with total spaces $\mathbb{C P}^{2} \# 13 \overline{\mathbb{C P}}^{2}, \mathrm{~K} 3 \# 2 \overline{\mathbb{C P}}^{2}$, and the Horikawa surface $H$, respectively.

For the Lefschetz fibrations with total spaces $\mathrm{K} 3 \# 2 \overline{\mathbb{C P}}^{2}$ and $H$, it is known that they have sections: The relation $\left(c_{1} c_{2} c_{3} c_{4} c_{5}\right)^{6}=\delta_{1} \delta_{2}$ in $\Gamma_{2,2}$ gives 2 disjoint 
sections for the Lefschetz fibration $\mathrm{K} 3 \# 2 \overline{\mathbb{C P}}^{2} \rightarrow S^{2}$, and $\left(c_{1} c_{2} c_{3} c_{4}\right)^{10}=\delta_{1}$ in $\Gamma_{2,1}$ gives a section for the Lefschetz fibration $H \rightarrow S^{2}$; see Figure 2.

Earlier, we found sections for the genus 2 Lefschetz fibration $\mathbb{C P}^{2} \# 13 \overline{\mathbb{C P}}^{2} \rightarrow S^{2}$. By sewing the sections during the fiber-sum operation, we get a section for any genus 2 holomorphic Lefschetz fibration without separating singular fibers.

Remark 5.3. One may continue and try to write similar relations for $9 \leq n \leq 12$ to see the exact number of disjoint sections that $\mathbb{C P}^{2} \# 13 \overline{\mathbb{C P}}^{2} \rightarrow S^{2}$ can admit. One can also try to find the exact number of disjoint sections of the genus 2 Lefschetz fibrations with total spaces $\mathrm{K} 3 \# 2 \overline{\mathbb{C P}}^{2}$ and $H$, respectively. It is still not known whether every genus $g$ Lefschetz fibration over $S^{2}$ admits a section.

\section{Appendix}

In this appendix, we deduce the relation

$$
\left(a_{1} b_{1} a_{2} b_{2}\right)^{5}=\left(a_{1} b_{1} a_{2}\right)^{4}\left(b_{2} a_{2} b_{1} a_{1}^{2} b_{1} a_{2} b_{2}\right),
$$

used in Section 4.1; for the corresponding curves, see Figure 4.

Note that $a_{1}$ intersects $b_{1}$ transversely at a single point, and commutes with $a_{2}$ and $b_{2}$. Also note that $a_{2}$ intersects $b_{1}$ and $b_{2}$ transversely at a single point, and the simple closed curves $b_{1}$ and $b_{2}$ commute. By the commutativity and braid relations, we have

$$
\begin{aligned}
\left(a_{1} b_{1} a_{2} b_{2}\right)^{5} & =\left(a_{1} b_{1} a_{2}\left(b_{2}\right)\right)\left(a_{1} b_{1} a_{2} b_{2}\right)\left(a_{1} b_{1} a_{2} b_{2}\right)^{3} \\
& =\left(a_{1} b_{1} a_{2}\right) a_{1} b_{1}\left(b_{2} a_{2} b_{2}\right)\left(a_{1} b_{1} a_{2} b_{2}\right)^{3} \\
& =\left(a_{1} b_{1} a_{2}\right) a_{1} b_{1}\left(a_{2} b_{2} a_{2}\right)\left(a_{1} b_{1} a_{2} b_{2}\right)^{3} \\
& =\left(a_{1} b_{1} a_{2}\right)^{2} b_{2} a_{2}\left(\left(a_{1}\right) b_{1} a_{2} b_{2}\right)\left(a_{1} b_{1} a_{2} b_{2}\right)^{2} \\
& =\left(a_{1} b_{1} a_{2}\right)^{2} a_{1} b_{2}\left(a_{2} b_{1} a_{2}\right) b_{2}\left(a_{1} b_{1} a_{2} b_{2}\right)^{2} \\
& =\left(a_{1} b_{1} a_{2}\right)^{2} a_{1} b_{2}\left(\left(b_{1}\right) a_{2}\left(b_{1}\right)\right) b_{2}\left(a_{1} b_{1} a_{2} b_{2}\right)^{2} \\
& =\left(a_{1} b_{1} a_{2}\right)^{2} a_{1} b_{1}\left(b_{2} a_{2} b_{2}\right)\left(b_{1} a_{1} b_{1}\right) a_{2} b_{2}\left(a_{1} b_{1} a_{2} b_{2}\right) \\
& =\left(a_{1} b_{1} a_{2}\right)^{2} a_{1} b_{1}\left(a_{2} b_{2} a_{2}\right)\left(a_{1} b_{1} a_{1}\right) a_{2} b_{2}\left(a_{1} b_{1} a_{2} b_{2}\right) \\
& =\left(a_{1} b_{1} a_{2}\right)^{3} b_{2} a_{2}\left(\left(a_{1}\right) b_{1} a_{1}\right)\left(a_{2}\right) b_{2}\left(a_{1} b_{1} a_{2} b_{2}\right) \\
& =\left(a_{1} b_{1} a_{2}\right)^{3} a_{1} b_{2}\left(a_{2} b_{1} a_{2}\right) a_{1} b_{2}\left(a_{1} b_{1} a_{2} b_{2}\right) \\
& =\left(a_{1} b_{1} a_{2}\right)^{3} a_{1} b_{2}\left(\left(b_{1}\right) a_{2} b_{1}\right) a_{1}\left(b_{2}\right)\left(a_{1} b_{1} a_{2} b_{2}\right) \\
& =\left(a_{1} b_{1} a_{2}\right)^{3} a_{1} b_{1}\left(b_{2} a_{2} b_{2}\right) b_{1} a_{1}\left(a_{1} b_{1} a_{2} b_{2}\right) \\
& =\left(a_{1} b_{1} a_{2}\right)^{3} a_{1} b_{1}\left(a_{2} b_{2} a_{2}\right) b_{1} a_{1}\left(a_{1} b_{1} a_{2} b_{2}\right) \\
& =\left(a_{1} b_{1} a_{2}\right)^{4}\left(b_{2} a_{2} b_{1} a_{1}^{2} b_{1} a_{2} b_{2}\right) .
\end{aligned}
$$




\section{Acknowledgments}

I am grateful to Anar Akhmedov for his suggestions and interest in this work. I would like to thank Kenneth L. Baker, John B. Etnyre, and Mustafa Korkmaz for helpful comments on the paper.

\section{References}

[Auroux 2003] D. Auroux, "Monodromy invariants in symplectic topology", preprint, 2003. arXiv math.SG/0304113

[Chakiris 1978] K. Chakiris, The monodromy of genus 2 pencils, thesis, Columbia University, 1978.

[Donaldson 1999] S. K. Donaldson, "Lefschetz pencils on symplectic manifolds", J. Differential Geom. 53:2 (1999), 205-236. MR 2002g:53154 Zbl 1040.53094

[Farb and Margalit 2005] B. Farb and D. Margalit, "A primer on mapping class groups", preprint, 2005, available at http://www.math.utah.edu/ margalit/primer/.

[Gervais 2001] S. Gervais, "A finite presentation of the mapping class group of a punctured surface", Topology 40:4 (2001), 703-725. MR 2002m:57025 Zbl 0992.57013

[Gompf and Stipsicz 1999] R. E. Gompf and A. I. Stipsicz, 4-manifolds and Kirby calculus, Graduate Studies in Mathematics 20, American Mathematical Society, Providence, RI, 1999. MR 2000h: 57038 Zbl 0933.57020

[Ivanov 2002] N. V. Ivanov, "Mapping class groups", pp. 523-633 in Handbook of geometric topology, edited by R. J. Daverman and R. B. Sher, North-Holland, Amsterdam, 2002. MR 2003h:57022 Zbl 1002.57001

[Korkmaz and Ozbagci 2008] M. Korkmaz and B. Ozbagci, "On sections of elliptic fibrations", Michigan Math. J. 56:1 (2008), 77-87. MR 2009f:57043 Zbl 1158.57033

[Smith 1998] I. Smith, Symplectic geometry of Lefschetz fibrations, thesis, University of Oxford, 1998.

Received October 4, 2009.

SINEM ÇELIK ONARAN

DEPARTMENT OF MATHEMATICS

MidDlE EAST TECHNICAL UNIVERSITY

ANKARA

TURKEY

e114485@metu.edu.tr

and

SCHOOL OF MATHEMATICS

GeORgIA InSTITUTE of TECHNOLOGY

ATLANTA, GA

UNITED STATES

sinemo@math.gatech.edu 


\title{
PACIFIC JOURNAL OF MATHEMATICS
}

\author{
http://www.pjmath.org \\ Founded in 1951 by \\ E. F. Beckenbach (1906-1982) and F. Wolf (1904-1989)
}

\section{EDITORS}

V. S. Varadarajan (Managing Editor)

Department of Mathematics

University of California

Los Angeles, CA 90095-1555

pacific@math.ucla.edu

Vyjayanthi Chari

Department of Mathematics

University of California

Riverside, CA 92521-0135

chari@math.ucr.edu

Robert Finn

Department of Mathematics Stanford University

Stanford, CA 94305-2125

finn@math.stanford.edu

Kefeng Liu

Department of Mathematics

University of California

Los Angeles, CA 90095-1555

liu@math.ucla.edu
Darren Long

Department of Mathematics

University of California

Santa Barbara, CA 93106-3080

long@math.ucsb.edu

Jiang-Hua Lu

Department of Mathematics

The University of Hong Kong

Pokfulam Rd., Hong Kong jhlu@maths.hku.hk

Alexander Merkurjev

Department of Mathematics

University of California

Los Angeles, CA 90095-1555

merkurev@math.ucla.edu
Sorin Popa

Department of Mathematics University of California

Los Angeles, CA 90095-1555 popa@math.ucla.edu

Jie Qing

Department of Mathematics

University of California

Santa Cruz, CA 95064

qing@cats.ucsc.edu

Jonathan Rogawski

Department of Mathematics

University of California

Los Angeles, CA 90095-1555

jonr@math.ucla.edu

\section{PRODUCTION}

pacific@math.berkeley.edu

\begin{abstract}
Silvio Levy, Scientific Editor Matthew Cargo, Senior Production Editor
\end{abstract}
ACADEMIA SINICA, TAIPEI

CALIFORNIA INST. OF TECHNOLOGY

INST. DE MATEMÁTICA PURA E APLICADA

KEIO UNIVERSITY

MATH. SCIENCES RESEARCH INSTITUTE

NEW MEXICO STATE UNIV.

OREGON STATE UNIV.

\section{SUPPORTING INSTITUTIONS}

STANFORD UNIVERSITY
UNIV. OF BRITISH COLUMBIA
UNIV. OF CALIFORNIA, BERKELEY
UNIV. OF CALIFORNIA, DAVIS
UNIV. OF CALIFORNIA, LOS ANGELES
UNIV. OF CALIFORNIA, RIVERSIDE
UNIV. OF CALIFORNIA, SAN DIEGO
UNIV. OF CALIF., SANTA BARBARA

UNIV. OF CALIF., SANTA CRUZ

UNIV. OF MONTANA

UNIV. OF OREGON

UNIV. OF SOUTHERN CALIFORNIA

UNIV. OF UTAH

UNIV. OF WASHINGTON

WASHINGTON STATE UNIVERSITY

These supporting institutions contribute to the cost of publication of this Journal, but they are not owners or publishers and have no responsibility for its contents or policies.

See inside back cover or www.pjmath.org for submission instructions.

The subscription price for 2010 is US \$420/year for the electronic version, and \$485/year for print and electronic.

Subscriptions, requests for back issues from the last three years and changes of subscribers address should be sent to Pacific Journal of Mathematics, P.O. Box 4163, Berkeley, CA 94704-0163, U.S.A. Prior back issues are obtainable from Periodicals Service Company, 11 Main Street, Germantown, NY 12526-5635. The Pacific Journal of Mathematics is indexed by Mathematical Reviews, Zentralblatt MATH, PASCAL CNRS Index, Referativnyi Zhurnal, Current Mathematical Publications and the Science Citation Index.

The Pacific Journal of Mathematics (ISSN 0030-8730) at the University of California, c/o Department of Mathematics, 969 Evans Hall, Berkeley, CA 94720-3840, is published monthly except July and August. Periodical rate postage paid at Berkeley, CA 94704, and additional mailing offices. POSTMASTER: send address changes to Pacific Journal of Mathematics, P.O. Box 4163, Berkeley, CA 94704-0163.

PJM peer review and production are managed by EditFLOW ${ }^{\mathrm{TM}}$ from Mathematical Sciences Publishers.

PUBLISHED BY PACIFIC JOURNAL OF MATHEMATICS

at the University of California, Berkeley 94720-3840

A NON-PROFIT CORPORATION

Typeset in LATEX

Copyright $(\mathrm{C} 2010$ by Pacific Journal of Mathematics 


\section{PACIFIC JOURNAL OF MATHEMATICS}

Volume $248 \quad$ No. $1 \quad$ November 2010

An existence theorem of conformal scalar-flat metrics on manifolds with boundary 1

SÉRgio de Moura Almaraz

Parasurface groups

KHALID BOU-RABEE

Expressions for Catalan Kronecker products

Andrew A. H. Brown, Stephanie Van Willigenburg and Mike

ZABROCKI

Metric properties of higher-dimensional Thompson's groups

José BURILLO and SEAN CLEARY

Solitary waves for the Hartree equation with a slowly varying potential

KIRIL DATCHEV and IVAN VENTURA

Uniquely presented finitely generated commutative monoids

PEDRO A. GARCíA-SÁNCHEZ and IgnaCio OJEDA

The unitary dual of $p$-adic $\widetilde{\operatorname{Sp}(2)}$

MARCELA HANZER and IVAN MATIĆ

A Casson-Lin type invariant for links

ERIC HARPER and NIKOLAI SAVELIEV

Semiquandles and flat virtual knots

ALLISON HENRICH and SAM NELSON

Infinitesimal rigidity of polyhedra with vertices in convex position

IVAN IZMESTIEV and JEAN-MARC SCHLENKER

Robust four-manifolds and robust embeddings

VYACHESLAV S. KRUSHKAL

On sections of genus two Lefschetz fibrations

SINEM ÇELIK ONARAN

Biharmonic hypersurfaces in Riemannian manifolds

YE-LIN OU

Singular fibers and 4-dimensional cobordism group 